RUNNING HEAD: Personality and healthy eating

\title{
Can Personality Close the Intention/Behavior Gap for Healthy Eating? An Examination with the HEXACO Personality Traits
}

\author{
Lauren A. Monds ${ }^{1}$, Carolyn MacCann ${ }^{2}$, Barbara A. Mullan ${ }^{1,2}$, Cara Wong ${ }^{2}$, Jemma \\ Todd $^{1}$, Richard D. Roberts ${ }^{3}$
}

\footnotetext{
${ }^{1}$ School of Psychology and Speech Pathology, Curtin University, WA, 6102, Australia

${ }^{2}$ School of Psychology, The University of Sydney, NSW, 2006, Australia

${ }^{3}$ Educational Testing Service, Research \& Development, Princeton, USA
}

The final, definitive version of this paper has been published in Psychology, Health \& Medicine by Taylor \& Francis Online: http://www.tandfonline.com/doi/full/10.1080/13548506.2015.1112416 
RUNNING HEAD: Personality and healthy eating

\begin{abstract}
The aim of this study was to investigate the predictive and moderating effects of HEXACO personality factors, in addition to theory of planned behavior (TPB) variables, on fruit and vegetable consumption. American college students $(N=1036)$ from 24 institutions were administered the TPB, HEXACO and a self-reported fruit and vegetable consumption measure. The TPB predicted $11-17 \%$ of the variance in fruit and vegetable consumption, with greater variance accounted for in healthy-weight compared to overweight individuals. Personality did not significantly improve the prediction of behavior above TPB constructs; however, conscientiousness was a significant incremental predictor of intention in both healthy-weight and overweight/obese groups. While support was found for the TPB as an important predictor of fruit and vegetable consumption in students, little support was found for personality factors. Such findings have implications for interventions designed to target students at risk of chronic disease.
\end{abstract}

Key words: personality; HEXACO; theory of planned behavior; healthy eating; fruit and vegetables; overweight 
RUNNING HEAD: Personality and healthy eating

\section{Introduction}

The association between dietary factors and chronic diseases such as cancer and diabetes is well established (Willett, 1994). Evidence suggests that healthy eating, in particular, consuming fruit and vegetables (FV) can help to reduce the risk of chronic diseases (Dauchet, Amouyel, Hercberg, \& Dallongeville, 2006; WHO/FAO, 2003); however, few people meet FV consumption recommendations (Kothe, Mullan, \& Butow, 2012; WHO/FAO, 2003). College/University students have low rates of consumption (Collins \& Mullan, 2011; Ding, Mullan, \& Xavier, 2014) and perceive FV as unpalatable and prefer unhealthy alternatives (Collins \& Mullan, 2011; Kothe \& Mullan, 2011 ). Further, weight gain and obesity worldwide are increasing (WHO Consultation, 2000) and consumption of FV is inversely related to obesity (Kothe \& Mullan, 2014).

The Theory of Planned Behavior (TPB; Ajzen, 1991) posits that intentions are the immediate antecedent to behavior and the stronger the intention the more likely behaviour will be performed (Ajzen, 1991). The TPB includes three predictors of intention - attitude, subjective norm and perceived behavioral control (PBC). PBC is also expected to directly influence behavior, insofar as it is a proxy for actual behavioral control (Ajzen, 1991).

The TPB has been used successfully and extensively used to predict FV consumption (Allom \& Mullan, 2012; Lien, Lytle, \& Komro, 2002) explaining on average $34 \%$ of the variance in intention and $23 \%$ of the variance in behavior (Guillaumie, Godin, \& Vézina-Im, 2010). Despite the utility of the TPB, research has found that intentions do not always translate into behavior (Armitage \& Conner, 2001; Godin \& Kok, 1996) leaving an 'intention-behavior gap' (Sniehotta, Scholz, \& Schwarzer, 2005). This is particularly true for health behaviors that require endurance, inconvenience, discomfort or have negative immediate outcomes (McEachan, Conner, Taylor \& Lawton, 2011). 
RUNNING HEAD: Personality and healthy eating

Research applying the TPB to overweight/obese samples is limited and inconsistent. Most research has focused on intention only. In one study of overweight women, TPB variables did not predict intention (Gardner \& Hausenblas, 2004). In contrast, another found the TPB strongly predicted exercise intention in obese individuals (Boudreau \& Godin, 2007). Further, in individuals at risk of developing diabetes up to $76 \%$ of the variance in intention to eat a healthy diet was accounted for by TPB variables (Blue, 2007). Thus, it remains unclear as to whether the TPB predicts health intentions for overweight/obese individuals. There is also a need for research predicting actual dietary behavior in this group. Individual differences in personality may offer a way to further improve the prediction of behavior, and assist in the translation of intentions into behavior (e.g., Lochbaum et al., 2010). The dominant model of trait personality is the five factor model (Digman, 1990; Tupes \& Christal, 1992). Under this model, there are five broad personality domains: Extraversion (the tendency to be gregarious, energetic, and positive); Agreeableness (the tendency to be sympathetic, moral, and altruistic); Conscientiousness (the tendency to work hard, focus on detail and adhere to rules); Neuroticism (the tendency to feel anxious, depressed or overwhelmed); and Openness (the tendency to be open to new experiences and ideas and be interested in intellectual pursuits). Recent research has suggested a sixth domain (Ashton \& Lee, 2007; Lee \& Ashton, 2008). This domain is Honesty/Humility, and represents differences in the extent to which people are sincere, fair and modest rather than greedy, conceited, and self-centered. This six-factor model is called the Honesty/HumilityEmotionality-eXtraversion-Agreeableness-Conscientiousness-Openness (HEXACO).

Although some recent research has integrated personality theory with existing social cognitive models such as the TPB (Conner \& Abraham, 2001), the majority of this research has focused on exercise (MacCann, Todd, Mullan, \& Roberts, 2015). In a recent review, conscientiousness was found to moderate the intention-behavior relationship such that 
RUNNING HEAD: Personality and healthy eating

individuals were more likely to act on intentions if they were higher in conscientiousness (Ferguson, 2013). It was also found that the relationship between conscientiousness and behavior was mediated by TPB variables such as attitudes.

Personality has been linked with patterns of food consumption and obesity. Lower conscientiousness and openness and higher neuroticism and extraversion have been found to be consistently associated with obesity (Armon, Melamed, Shirom, Shapira, \& Berliner, 2013; Sutin, Ferrucci, Zonderman, \& Terracciano, 2011). Additionally, a meta-analysis revealed that conscientiousness predicts numerous health related behaviors including unhealthy eating ( $\rho=-.25$; Bogg \& Roberts, 2004). With regards to FV consumption de Bruijn, Kremers, van Mechelen, \& Brug (2005) found agreeableness and openness were associated with vegetable consumption, and openness was also associated with fruit consumption. Further, de Bruijn, Brug, \& Van Lenthe (2009) found that the conscientiousness-behavior relationship was mediated by TPB variables, and also that neuroticism moderated the intention-behavior relationship suggesting a relationship between TPB variables and personality domains in explaining FV consumption.

However, the abovementioned research did not use the more recent HEXACO personality model; the addition of the honesty/humility domain may further explain FV consumption behavior. Moreover, as very little research has attempted to integrate the effects of personality on food consumption within a TPB framework, this study examines the effects of HEXACO in predicting FV consumption in conjunction with TPB. Additionally, FV consumption in the USA is lowest amongst 15-29-year-olds (World Health Organisation, 2004). As the college/university lifestyle has been seen to influence the eating patterns of young adults (Leslie, Sparling, \& Owen, 2001) which means this is an important cohort to study regarding prediction of FV consumption. Therefore, examining whether personality 
RUNNING HEAD: Personality and healthy eating

factors can improve the prediction of FV consumption may inform interventions to improve FV consumption within students.

\section{Aims and Hypotheses}

The aim of this research was to explore the role of TPB and HEXACO in predicting FV consumption. It was expected that the TPB would predict intention, and that HEXACO domains would incrementally improve prediction of intention. It was also expected that the TPB would predict FV consumption, and that the HEXACO would further improve the prediction of behavior. Furthermore, HEXACO domains were expected to moderate the intention-behavior gap. The final and exploratory aim of this study was to compare healthyweight versus overweight/obese students on TPB and HEXACO constructs in predicting FV consumption.

\section{Methods}

\section{Participants}

Participants were 1036 students (63.9\% female) attending one of 24 institutions in the USA ( $n=574,64.5 \%$ female; see MacCann et al., 2015, for further details of the sample). Students ranged in age from 15 to $85(M=23.08, S D=7.434)$. Institutions were located in five different regions of the USA. Participants predominantly identified as Black (23.1\%), Hispanic (13.6\%), White (52.5\%), or Asian (5.6\%). Participants were classified as either underweight $(n=41)$, healthy-weight $(n=501)$, or overweight/obese $(n=475)$. Subsequent analyses compared healthy-weight individuals with the combined group of overweight/obese individuals.

\section{Procedure}

Participants were recruited through colleges, and tested in local testing centers (usually on the institution campus). Participants completed a two-hour computerized test 
RUNNING HEAD: Personality and healthy eating

battery which included demographic questions, self-reports of personality, and TPB questions regarding nutrition and exercise (for exercise data see MacCann et al., 2015). After testing, participants were reimbursed for their time. This testing protocol was approved by ETS fairness and the Human Research Ethics Committee of the participating institutions.

\section{Instrumentation}

The TPB components were assessed with 24 items on a 5-point Likert scale, from Strongly disagree to Strongly agree; attitude (e.g., "I like eating healthy food"), subjective norm (e.g., "Most people I know are careful about what they eat"), perceived behavioral control (e.g., "It's too much effort to follow a healthy diet" [reverse]) and intention (e.g., "I intend to follow a nutritious diet”). Cronbach's alphas ranged from .72 to .89 (Table 1). The HEXACO domains of personality were assessed using 96 items from the International Personality Item Pool (IPIP; Goldberg et al., 2006). Each of the six domains of personality were represented by 16 items: honesty/humility (e.g., "I tell the truth”); emotionality (e.g., "I have a dark outlook on the future”); extraversion (e.g., "I talk a lot”); agreeableness (e.g., "I am inclined to forgive others"); conscientiousness (e.g., "I like to plan ahead"); and openness to experience (e.g., "I enjoy contemplation”). Participants rated each item on a five-point scale, from very inaccurate to very accurate. Cronbach's alphas ranged from .77 to .86 (Table 1).

[Table 1 here]

Behavior was assessed through the frequency with which participants ate four types of FV: (1) green vegetables (e.g., broccoli, spinach,); (2) fresh fruit (e.g., apples, plums); (3) orange vegetables (e.g., carrots, pumpkin); and (4) Legumes (e.g., kidney-beans, chick-peas). 
RUNNING HEAD: Personality and healthy eating

Participants rated these statements on a 7-point Likert scale from: (1) Not at all to (7) More than once a day. Cronbach's alpha was .75.

\section{Results}

\section{Descriptive statistics and correlations}

Table 1 shows the means, standard deviations and Cronbach's alpha reliabilities for all TPB variables and HEXACO domains for normal weight versus overweight/obese. Groups did not differ significantly on any of the HEXACO domains, nor on subjective norm or FV consumption. Compared to the overweight/obese group, healthy-weight participants had more positive attitudes $(t=2.654 ; d f=974 ; p=.008)$, higher $\operatorname{PBC}(t=2.025 ; d f=974 ; p$ $=.043)$ and stronger intentions $(t=2.433 ; d f=974 ; p=.015)$. However, these differences were of small effect size (Hedge's $g=.17, .13$ and .16 respectively).

Correlations between TPB variables, HEXACO domains and FV consumption are also reported in Table 1 . Intention was significantly associated with attitude, subjective norm, and PBC for the total sample and for the overweight/obese group. In the healthy-weight group, intention was significantly associated with attitude and PBC but not with subjective norm. Attitude showed the strongest relationship to intention in all cases. Behavior was significantly related to both intention and PBC in the total sample as well as both groups.

Intention was significantly correlated with all HEXACO domains in the total sample, with the strongest relationship for Conscientiousness $(r=.28)$. Intention was also significantly correlated with all HEXACO domains in the healthy-weight group, and with all domains but Agreeableness in the overweight/obese group. HEXACO domains showed weaker correlations with behavior than with intention. For the whole sample, intention was significantly associated with conscientiousness, openness, emotionality and extraversion, but effect sizes were very small (.07 to .12). For the healthy-weight group, only openness 
RUNNING HEAD: Personality and healthy eating

significantly predicted behavior. For the overweight/obese group, conscientiousness, extraversion, emotionality, and openness significantly predicted behavior, with the strongest effect for conscientiousness $(r=.19)$.

\section{Prediction of Intention}

After controlling for demographic variables, TPB variables significantly improved the prediction of intention, explaining $34.6 \%$ of variance in the overall sample $(32.7 \%$ in healthy sample and $36.0 \%$ in overweight/ obese participants). Attitudes and subjective norm were significant independent predictors across all samples, with attitudes the strongest predictor.

Personality variables significantly improved prediction of intention, explaining an additional $3.0 \%$ of variance in the overall sample (4.3\% in healthy sample and $2.8 \%$ in overweight/obese participants). There were no significant differences in the amount of variance explained across the groups (Fisher's $z$ test was used to compare multiple correlations; $z=0.650 ; p>.05)$. For the overall sample, conscientiousness and openness were the only significant independent predictors. For the healthy-weight sample, honesty/humility and conscientiousness were significant independent predictors, whilst for the overweight and obese sample, conscientiousness and openness (but not honesty/humility) were significant independent predictors. The size of regression coefficients for healthy-weight compared overweight/obese participants was not significantly different for conscientiousness or openness but was significantly different for honesty/humility (Fisher's $z=2.45 ; p=.014$ ). That is, honesty/humility lead to stronger intention among healthy-weight individuals, but had no relationship to intention for overweight/obese individuals (see table 2).

\section{Prediction of Behavior}

After controlling for demographic variables, TPB variables of intention and PBC explained an additional $14.1 \%$ of the variance in FV consumption for the overall sample (17.2\% for the healthy-weight group and $10.9 \%$ for the overweight/obese group). This 
RUNNING HEAD: Personality and healthy eating

difference between groups was not significant using Fisher's $z$ test $(z=1.53 ; p=.126)$. Both PBC and intention were independently predictive of behavior for the whole sample, and for those of healthy-weight, but only intention was independently predictive of behavior for those who were overweight/obese. Moreover, intention was a significantly stronger predictor of behavior for the healthy-weight group than the overweight/obese group (Fisher's $z=2.33$; $p=.020)$.

HEXACO domain variables did not explain a significant amount of variance in behavior after TPB variables; however, honesty/humility was a significant independent predictor for the total sample and for those of healthy-weight, but not for those who were overweight/obese. None of the interaction terms were significant, indicating that personality did not moderate the effect of intentions on behavior in this study (see table 2).

[table 2 here]

\section{Discussion}

The present study adds to the evidence base that the TPB model is predictive of FV consumption (Guillaumie et al., 2010; Kothe \& Mullan, 2014; Kothe et al., 2012); and that personality domains (particularly conscientiousness) may predict FV intention. There was support for the TPB model as a predictor of FV consumption. The TPB explained $35 \%$ of intentions and $14 \%$ of behavior. However, these were lower than reported in previous research investigating dietary behaviors (50\% and 21\% respectively; McEachan et al., 2011). It is possible that using a geographically and culturally diverse sample, may account for these differences.

In addition, PBC did not significantly predict intention for either healthy-weight or overweight/obese groups in the current study, and also did not significantly predict behavior 
RUNNING HEAD: Personality and healthy eating

in the overweight/obese sample. This is inconsistent with previous research examining FV consumption in college students, where path analysis found that $\mathrm{PBC}$ predicted intention but not behavior (Blanchard et al., 2009). One possible difference from previous research is our conceptualization of PBC. It is known that PBC is a multi-dimensional construct that includes controllability as well as self-efficacy beliefs (Ajzen, 2002; Collins \& Mullan, 2011). In the current study, $\mathrm{PBC}$ items represented perceived barriers to FV consumption and therefore addressed only the controllability component of PBC. Previous research on exercise suggests that self-efficacy is a better predictor of behavior, whereas controllability is a better predictor of intention (Terry \& O'Leary, 1995). Results from the current study suggest that this differential prediction of intention and behavior by controllability and self-efficacy may also hold for FV consumption.

Predictors of intention and behavior were not significantly different across groups, suggesting that the TPB model is accurate in both samples. However, there were two key differences between groups: intention was a significantly stronger predictor of behavior for healthy-weight compared to overweight/obese individuals; and PBC significantly predicted intentions in the healthy-weight but not the overweight/obese group. Thus, while the TPB model predicts intention and behavior in both groups, evidence is weaker for the overweight/obese group than the healthy-weight group. It may be that there are additional factors of importance for overweight/obese individuals when making healthy eating choices or that such behavior is less under the volitional control of overweight/obese individuals (Robertson, Mullan \& Todd, 2014). Given that interventions to increase FV consumption are needed for overweight/obese individuals such differences have important implications. For example, an intervention designed and tested on a healthy-weight group may be less effective in an overweight/obese group due to the weaker association between intention and behavior. Such results point to the importance of using targeted samples in research on the TPB. 
RUNNING HEAD: Personality and healthy eating

There was little support for the HEXACO domains as incremental predictors of FV consumption. Although personality domains added significantly to the prediction of intention, the effect size was small (3\% of the variance) and personality did not incrementally predict behavior, nor moderate the relationship between intention and behavior. Conscientiousness was a significant predictor of intention for all groups. This is in accordance with previous literature that has found conscientiousness predictive of health protective intention (Conner \& Abraham, 2001), and suggests that those who are more organized and disciplined towards their goals are more likely to intend to eat healthily. No other personality domain showed a consistent significant effect across groups.

Openness was a small but significant predictor of FV consumption intention in those who were overweight/obese. This is in contrast to previous research, which has generally not found openness to be predictive in health (Ferguson, 2013), although it is consistent with de Bruijn, Kremers, van Mechelen and Brug (2005) who found a similar positive relationship for FV consumption. Although this finding should not be over-interpreted it is possible that those who are overweight and higher on openness may be able to consider behavior changes, and further research could explore these associations.

Personality did not significantly add to the prediction of FV consumption. Honesty/humility emerged as a significant independent predictor, but only for those of healthy-weight, and no other predictors were significant. That those who were high in honesty/humility within the healthy-weight sample were more likely to set healthy intentions (but less likely to act on these intention) was unexpected; but suggests that those who feel no special entitlement or desire for lavish possessions and are also healthy are more likely to continue to intend to consume FV, or are perhaps more honest in their reporting of their actual FV consumption, reflecting a more accurate behavior record than those who were lower in honesty/humility, who may be more motivated towards social desirability and over- 
RUNNING HEAD: Personality and healthy eating

reporting. However, given the size of the finding, these results need to be interpreted with caution and warrant further investigation.

\section{Limitations and Strengths}

The current study was conducted cross-sectionally and self-report was used; these are potential limitations of the study only subjective reports of FV consumption behavior over the one time period were captured rather than over the long term. However, this design allowed for a large and diverse sample to be recruited. As some of the correlations and variances explained in the regressions were modest, this suggests other relevant variables or confounding factors were missing from the study; for example, availability and price of FV may be important as well as attitudes relating to preparing them for consumption. These factors may be worth considering for future research. One strength of the study was the methodological rigor of the questionnaire design. Where many studies in this area use single measures to assess constructs, multiple measures were used in the current study, and the HEXACO model of personality was chosen because of its psychometric validity.

\section{Conclusions}

This study provided support for the TPB as a model for predicting FV consumption across a large and diverse sample. TPB can be used with overweight/obese samples, though with potentially smaller effects than healthy-weight participants. Further research should investigate whether TPB-informed weight-loss interventions are effective. Despite the intention-behavior gap, personality added only a small amount to the prediction of intention, but not to behavior. Whilst conscientiousness appears to be important in forming an intention, it does not appear to influence actual behavioral engagement. Although personality could be 
RUNNING HEAD: Personality and healthy eating

influential in forming beliefs, it does not appear necessary to tailor interventions based on TPB variables to different personalities. 
RUNNING HEAD: Personality and healthy eating

\section{References}

Ajzen, I. (1991). The theory of planned behaviour. Organizational Behavior and Human Decision Processes, 50, 179-211. doi: 10.1016/0749-5978(91)90020-T

Ajzen, I. (2002). Perceived behavioral control, self-efficacy, locus of control, and the theory of planned behavior. Journal of Applied Social Psychology, 32(4), 665-683. doi: 10.1111/j.1559-1816.2002.tb00236.x

Allom, V., \& Mullan, B. (2012). Self-regulation versus habit: The influence of self-schema on fruit and vegetable consumption. Psychology \& Health, 27(Supp 2), 7-24. doi: $10.1080 / 08870446.2011 .605138$

Armitage, C., \& Conner, M. (2001). Efficacy of the theory of planned behaviour: A metaanalytic review. British Journal of Social Psychology, 40, 471-499. doi: $10.1348 / 014466601164939$

Armon, G., Melamed, S., Shirom, A., Shapira, I., \& Berliner, S. (2013). Personality traits and body weight measures: Concurrent and across time associations. European Journal of Personality, 27(4), 398-408. doi: 10.1002/per.1902

Ashton, M. C., \& Lee, K. (2007). Empirical, theoretical, and practical advantages of the HEXACO model of personality structure. Personality and Social Psychology Review, 11(2), 150-166. doi: 10.1177/1088868306294907

Blanchard, C. M., Kupperman, J., Sparling, P. B., Nehl, E., Rhodes, R. E., Courneya, K. S., \& Baker, F. (2009). Do ethnicity and gender matter when using the theory of planned behavior to understand fruit and vegetable consumption? Appetite, 52(1), 15-20. doi: 10.1016/j.appet.2008.07.001

Blue, C. L. (2007). Does the theory of planned behavior identify diabetes-related cognitions for intention to be physically active and eat a healthy diet? Public Health Nursing, 24(2), 141-150. doi: 10.1111/j.1525-1446.2007.00618.x 
RUNNING HEAD: Personality and healthy eating

Bogg, T., \& Roberts, B. W. (2004). Conscientiousness and health-related behaviors: A metaanalysis of the leading behavioral contributors to mortality. Psychological Bulletin, 130(6), 887-919. doi: 10.1037/0033-2909.130.6.887

Boudreau, F., \& Godin, G. (2007). Using the theory of planned behaviour to predict exercise intention in obese adults. Canadian Journal of Nursing Research, 39(2), 112-125.

Collins, A., \& Mullan, B. (2011). An extension of the theory of planned behavior to predict immediate hedonic behaviors and distal benefit behaviors. Food Quality and Preference, 22(7), 638-646. doi: http://dx.doi.org/10.1016/j.foodqual.2011.03.011

Conner, M., \& Abraham, C. (2001). Conscientiousness and the theory of planned behavior: Toward a more complete model of the antecedents of intentions and behavior. Personality and Social Psychology Bulletin, 27(11), 1547-1561. doi: $10.1177 / 01461672012711014$

Dauchet, L., Amouyel, P., Hercberg, S., \& Dallongeville, J. (2006). Fruit and vegetable consumption and risk of coronary heart disease: a meta-analysis of cohort studies. The Journal of nutrition, 136(10), 2588-2593.

de Bruijn, G.-J., Brug, J., \& Van Lenthe, F. J. (2009). Neuroticism, conscientiousness and fruit consumption: Exploring mediator and moderator effects in the theory of planned behaviour. Psychology and Health, 24(9), 1051-1069.

de Bruijn, G.-J., Kremers, S. P. J., van Mechelen, W., \& Brug, J. (2005). Is personality related to fruit and vegetable intake and physical activity in adolescents? Health Education Research, 20(6), 635-644. doi: 10.1093/her/cyh025

Digman, J. M. (1990). Personality structure: Emergence of the five-factor model. Annual Review of Psychology, 41(1), 417-440. doi: 10.1146/annurev.ps.41.020190.002221 
RUNNING HEAD: Personality and healthy eating

Ding, T., Mullan, B., \& Xavier, K. (2014). Does subjective well-being predict healthenhancing behaviour? The example of fruit and vegetable consumption. British Food Journal, 116(4), 598-610.

Ferguson, E. (2013). Personality is of central concern to understand health: Towards a theoretical model for health psychology. Health Psychology Review, 7(sup1), S32S70. doi: 10.1080/17437199.2010.547985

Gardner, R. E., \& Hausenblas, H. A. (2004). Understanding Exercise and Diet Motivation in Overweight Women Enrolled in a Weight-Loss Program: A Prospective Study Using the Theory of Planned Behavior. Journal of Applied Social Psychology, 34(7), 13531370. doi: 10.1111/j.1559-1816.2004.tb02010.x

Godin, G., \& Kok, G. (1996). The theory of planned behavior: A review of its applications to health-related behaviors. American Journal of Health Promotion, 11(2), 87-98. doi: $10.4278 / 0890-1171-11.2 .87$

Guillaumie, L., Godin, G., \& Vézina-Im, L.-A. (2010). Psychosocial determinants of fruit and vegetable intake in adult population: A systematic review. International Journal of Behavioral Nutrition and Physical Activity, 7(1), 12. doi: 10.1186/1479-5868-7-12

Kothe, E., \& Mullan, B. (2011 ). Perceptions of fruit and vegetable dietary guidelines amongst Australian young adults. Nutrition \& Dietetics(68), 262-266. doi: http://dx.doi.org/10.1111/j.1747-0080.2011.01550.x

Kothe, E., \& Mullan, B. (2014). A randomised controlled trial of a theory of planned behaviour to increase fruit and vegetable consumption: Fresh Facts. Appetite, 78, 6875.

Kothe, E., Mullan, B., \& Butow, P. (2012). Promoting fruit and vegetable consumption: Testing an intervention based on the theory of planned behaviour. Appetite, 58(3), 997-1004. doi: http://dx.doi.org/10.1016/j.appet.2012.02.012 
RUNNING HEAD: Personality and healthy eating

Lee, K., \& Ashton, M. C. (2008). The HEXACO personality factors in the indigenous personality lexicons of English and 11 other languages. Journal of Personality, 76(5), 1001-1053.

Leslie, E., Sparling, P. B., \& Owen, N. (2001). University campus settings and the promotion of physical activity in young adults: lessons from research in Australia and the USA. Health Education, 101(3), 116-125.

Lien, N., Lytle, L. A., \& Komro, K. A. (2002). Applying Theory of Planned Behavior to Fruit and Vegetable Consumption of Young Adolescents. American Journal of Health Promotion, 16(4), 189-197. doi: 10.4278/0890-1171-16.4.189

Lochbaum, M. R., Rhodes, R. E., Stevenson, S. J., Surles, J., Stevens, T., \& Wang, C. K. J. (2010). Does gender moderate the exercising personality? An examination of continuous and stage-based exercise. Psychology, Health \& Medicine, 15(1), 50-60. doi: $10.1080 / 13548500903443449$

MacCann, C., Todd, J., Mullan, B. A., \& Roberts, R. D. (2015). Can personality bridge the intention-behavior gap to predict who will exercise? Am J Health Behav, 39(1), 140147. doi: 10.5993/ajhb.39.1.15

McEachan, R. R. C., Conner, M., Taylor, N. J., \& Lawton, R. J. (2011). Prospective prediction of health-related behaviours with the Theory of Planned Behaviour: a meta-analysis. Health Psychology Review, 5(2), 97-144. doi: $10.1080 / 17437199.2010 .521684$

Robertson, A., Mullan, B., \& Todd, J. (2014). A Qualitative Exploration of Experiences of Overweight Young and Older Adults: An Application of the Theory of Planned Behaviour. Appetite, 75, 157-164

Sniehotta, F., Scholz, U., \& Schwarzer, R. (2005). Bridging the intention-behaviour gap: Planning, self-efficacy, and action control in the adoption and maintenance of 
RUNNING HEAD: Personality and healthy eating

physical exercise. Psychology \& Health, 20(2), 143-160. doi:

$10.1080 / 08870440512331317670$

Sutin, A. R., Ferrucci, L., Zonderman, A. B., \& Terracciano, A. (2011). Personality and obesity across the adult life span. Journal of Personality and Social Psychology, 101(3), 579-592. doi: 10.1037/a0024286

Terry, D. J., \& O'Leary, J. E. (1995). The theory of planned behaviour: The effects of perceived behavioural control and self-efficacy. British Journal of Social Psychology, 34(2), 199-220. doi: 10.1111/j.2044-8309.1995.tb01058.x

Tupes, E. C., \& Christal, R. E. (1992). Recurrent personality factors based on trait ratings. Journal of Personality, 60(2), 225-251. doi: 10.1111/j.1467-6494.1992.tb00973.x

WHO Consultation. (2000). Obesity: preventing and managing the global epidemic World Health Organization Technical Report Series (Vol. 894).

WHO/FAO. (2003). Diet, nutrition and the prevention of chronic diseases Technical report series 916. Geneva: World Health Organization.

Willett, W. C. (1994). Diet and health: What should we eat? Science, 264(5158), 532-537. doi: $10.1126 /$ science. 8160011

World Health Organisation. (2004). Comparative quantification of health risks: Global and regional burden of disease attributable to selected major risk factors. Geneva: World Health Organisation. 